\title{
Dashboard para Gestão Acadêmica
}

\author{
Robson Ferreira Gomes ${ }^{1}$, José Henrique Coelho Brandão ${ }^{1}$, Fernanda Pereira Gomes ${ }^{1}$, \\ Parcilene Fernandes de Brito ${ }^{1}$ \\ ${ }^{1}$ Departamento de Computação - Centro Universitário Luterano de Palmas \\ (CEULP/ULBRA) Avenida Teotônio Segurado, CEP 77.019-900 - Palmas - TO - \\ Brasil \\ \{fgomes.robson, jhcb007, fernandagomes 769, parcilene\}@gmail.com
}

\begin{abstract}
Resumo. Dashboards são ferramentas úteis para apoiar a tomada de decisão em organizações, devido a sua eficácia na apresentação dos dados, foco nos objetivos a serem alcançados e recursos que proporcionam interatividade aos gestores na exploração dos dados. Neste sentido, o presente trabalho propõe a criação de um dashboard voltado para o contexto da Gestão Acadêmica, mais especificamente as atividades relacionadas ao contexto do docente. Para que fosse possivel o desenvolvimento do dashboard, foram criados mecanismos (scripts) para que a coleta de dados provenientes de outra plataforma pudesse ser realizada de forma automática.
\end{abstract}

\section{Introdução}

As frequentes melhorias na área da Tecnologia da Informação têm permitido o constante acúmulo de grandes volumes de dados armazenados. Segundo Goldschmidt e Bezerra (2017) diversas tecnologias, tais como internet, sistemas gerenciadores de banco de dados, leitores de códigos de barra e sistemas de informação em geral são alguns exemplos de recursos que têm viabilizado o aumento no volume de dados armazenados de natureza comercial, administrativa, governamental e científica.

A análise desse grande volume de dados de forma manual se torna inviável, visto que, a quantidade de tempo e esforços gastos para tal tarefa seria muito alta. Portanto, é de suma importância a criação ou utilização de técnicas ágeis, inteligentes e que permitam transformar essa enorme quantidade de dados em informações úteis e relevantes em determinados contextos.

Nesse sentido, os dashboards podem oferecer uma solução para o problema de sobrecarga de informação, facilitando a tarefa de gestão e auxiliando no processo de tomada de decisão. O dashboard proposto no presente trabalho é aplicado ao contexto da Gestão Acadêmica, e foram considerados os dados provenientes de um sistema web utilizado por coordenadores, professores e alunos do Centro Universitário Luterano de Palmas CEULP/ULBRA.

Seu desenvolvimento deu-se em etapas distintas, desde reuniões realizadas com a especialista de domínio, estudo bibliográfico sobre técnicas eficazes na criação de ferramentas dashboard, em que foram verificadas boas práticas na criação e validação de protótipos funcionais, até a construção e implantação do dashboard proposto.

\section{Dashboard}

Few (2006) afirma que dashboards são exibições visuais de informações importantes, organizadas e apresentadas em uma única tela de forma que um ou mais objetivos possam ser alcançados. Para o autor, um dashboard ou "painel de controle" é um produto de software de fácil uso e entendimento que fornece uma representação gráfica, condensada de informações qualitativas ou quantitativas.

Segundo Buchsbaum (2012) dashboards devem representar informações visuais exibidas em uma única tela, além disso, possuem numerosas formas de interação com o usuário que servem como um ponto inicial para as demais informações presentes em uma organização. Já Duarte (2012) explica que dashboards podem ser considerados sistemas de 
apoio à decisão, uma vez que sua interface gráfica abriga indicadores de performance de um negócio, possibilitando a tarefa de gestão e servindo de apoio a tomada de decisão. Em uma síntese apresentada por Yigitbasioglu e Velcu (2012, p.4)

"Dashboard é uma ferramenta de gerenciamento de desempenho visual e interativo que exibe em uma única tela as informações mais importantes necessárias para atingir uma ou várias metas individuais e/ou organizacionais, permitindo ao usuário identificar, explorar e comunicar áreas problemáticas que precisam de ação corretiva." (Yigitbasioglu e Velcu, 2012, p.4).

O sucesso de um dashboard está intimamente ligado à sua capacidade de exibir informações relevantes para um determinado contexto, uma vez que se torna comum sua utilização por diferentes pessoas ocupando diferentes cargos dentro das instituições. Segundo Pauwels et al. (2009), os gestores mencionam alguns fatores que determinam a necessidade do uso de dashboards, que vão desde à dificuldade em organização de muitos dados que sejam potencialmente relevantes para tomada de decisões estratégicas, demandas crescentes de informações para as diversas áreas da empresa, até a necessidade de integração entre os diversos setores de uma organização nas práticas de relatos de desempenho, alocação de recursos, dentre outros.

Few (2006) explica que dentre as inúmeras formas existentes para categorizar dashboards, a maneira como seus elementos visuais se comportam podem ser usados para definir seus vários tipos. Segundo ele, os tipos clássicos de design de dashboards estão subdivididos em 3 grandes grupos: estratégicos, analíticos e operacionais.

Os dashboards estratégicos concentram-se em exibir indicadores de desempenho que auxiliem os gerentes a tomar decisões que podem impactar diretamente no futuro da organização. Já os dashboards analíticos, para que seja possível estabelecer uma relação de causa e efeito nos dados é necessário o uso de meios de exibição mais sofisticados para que o analista possa entender não só o resultado final, mas também as transformações que os dados sofreram até chegar ao conteúdo apresentado na tela. Por fim, os dashboards operacionais devem ser projetados de forma diferente dos dashboards estratégicos e analíticos, pois sua natureza dinâmica e sua alta frequência de uso (diário) torna necessário a atualização de dados em tempo real (FEW, 2006).

$\mathrm{O}$ formato de apresentação dos dados permite que o gestor concentre-se em informações importantes rapidamente, tais como: o momento em que o fluxo de acessos aumenta ou diminui, principais páginas acessadas e países com maior número de acessos. Em posse deste tipo de informação, o gestor pode tomar medidas corretivas capazes de assegurar que tarefas rotineiras de uma organização possam seguir seu curso livremente, dessa forma, prevenindo a ocorrência de falhas.

Few (2006) explica que a maior parte dos dashboards expressam principalmente medidas quantitativas, pois este tipo de dados permite monitorar as informações necessárias para atender um ou mais objetivos específicos. Segundo ele, as principais formas de expressar dados em dashboards são: dados não quantitativos, enriquecimento por comparação, e enriquecimento por avaliação.

Segundo Few (2006), os dados não quantitativos são uma ótima alternativa para apresentação de indicadores de performance em dashboards. Já no enriquecimento por comparação é comum apresentar as métricas definidas de forma individual, entretanto a comparação com métricas correlacionadas ajudar a fornecer um contexto maior e assim aumentar ainda mais sua relevância e significado. Para Buchsbaum (2012) as métricas de um dashboard devem representar um conjunto de indicadores capazes de refletir os principais objetivos da organização, e estas podem ser coletadas a partir de perguntas direcionadas a um gestor ou especialista de domínio. 
Por fim, no enriquecimento por avaliação, surge como uma alternativa viável para exibição de grandes volumes de dados de forma que a percepção do usuário não seja prejudicada, sua utilidade se concentra na capacidade de classificar rapidamente determinado conjunto de dados em categorias como "bom" ou "ruim", "alto" ou "baixo", e etc (FEW, 2006). Para isso, elementos visuais simples podem ser codificados (como por exemplo, um indicador preenchido com a cor verde para indicar um situação favorável dos dados) para que o usuário possa identificar eventos relevantes e potencialmente úteis dado determinado contexto.

\subsection{Arquitetura}

A arquitetura de um dashboard permite a definição de seus componentes e facilita a comunicação entre os interessados no software. Staron (2015) explica que o modelo de arquitetura subdivido em camadas é o mais utilizado para o desenvolvimento de dashboards, uma vez que permite o processamento de informações em formato de fluxo, ou seja, sem a necessidade de todas as camadas estarem ligadas diretamente umas às outras. A Figura 1, apresenta um modelo de arquitetura proposto por Staron (2015).

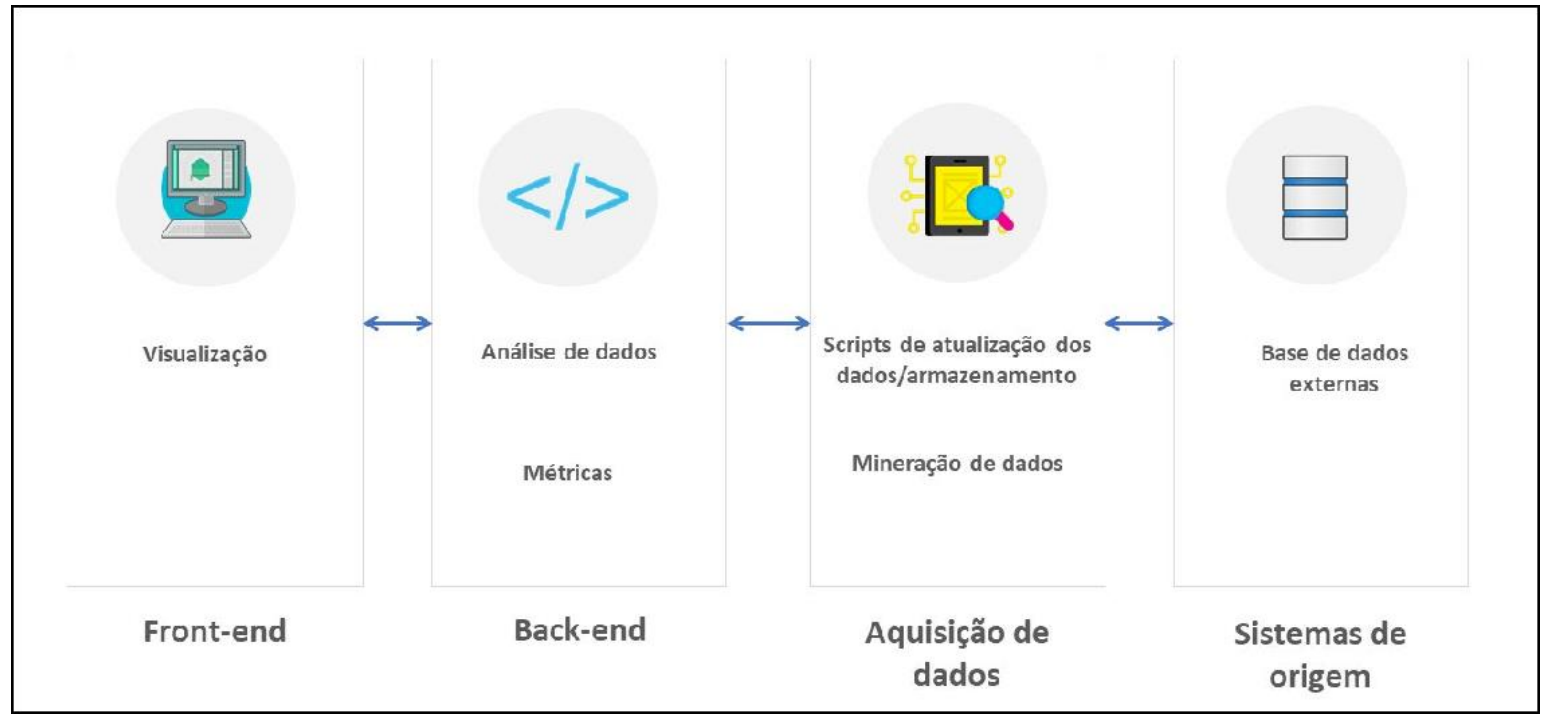

Figura 1. Modelo de arquitetura de um dashboard

O Front-end representa a interface de interação com o usuário. Para Staron (2015) por mais que o Front-end da aplicação seja o elemento mais perceptível ao usuário, o mesmo não deve ser considerado o componente principal da arquitetura, uma vez que o Back-end é o principal responsável pelo processamento das métricas a serem exibidas na tela do dashboard.

O Back-end da aplicação é responsável por todo o processamento de dados necessário a visualização (STARON, 2015). Nesta camada também podem ser realizadas análises com o objetivo de agregar mais conhecimento sobre os dados armazenados. Staron (2015) explica ainda que a camada de Aquisição de dados contém scripts e softwares responsáveis por reunir os dados necessários à aplicação, provenientes de múltiplos sistemas de origem.

Esta camada é de suma importância para o processo de desenvolvimento de um dashboard, uma vez que é nela onde ocorre a coleta, sumarização e armazenamentos dos dados selecionados. Por fim, os Sistemas de origem visam fornecer os dados necessários ao funcionamento do dashboard. Segundo Staron (2015) os sistemas de origem fazem parte do dia a dia da organização e provêm bancos de dados nos quais os dados podem ser coletados.

\section{Materiais e Métodos}


Para o desenvolvimento do presente trabalho, foi utilizada a base de dados do Conecta, e do módulo Coordenação. O primeiro consiste em uma plataforma com o objetivo de apoiar atividades acadêmicas, visando a troca de informações entre professores e alunos, e o segundo contém informações de eixos, cursos, alunos, professores, turmas, registro de conteúdo, registro de frequência, registro de reuniões, registro de falta e reposições dos professores, registro de turmas abaixo da média e registro de problemas em orientações.

\subsection{Materiais}

Para o desenvolvimento do dashboard proposto, foram utilizados diversos recursos e ferramentas de software, das quais pode-se destacar:

- PHP: Uma das linguagens para programação WEB mais utilizadas no mundo, devido ao seu pouco consumo de memória, velocidade e ser tolerante a falhas por tratar cada processo isolado, assim se ocorrer falha em alguma solicitação, não afetará todo o sistema.

- Silex: Um micro framework PHP para pequenas aplicações com foco na agilidade, extensibilidade e para ser facilmente testável.

- Javascript: Uma linguagem de programação interpretada utilizada para controlar em tempo de execução o HTML e o CSS, permitindo desenvolver funcionalidades mais complexas em página web.

- AngularJS: Framework para Javascript que viabiliza a criação de aplicações WEB dinâmicas, simplificando a codificação e estruturando o código no padrão MVC.

\subsection{Métodos}

O resultado esperado é um dashboard (software) que apresente informações de forma clara, distinta e de mais rápida assimilação pelo usuário, permitindo auxiliar no processo de tomada de decisão. Para o desenvolvimento do dashboard proposto foram especificadas algumas etapas, que vão desde entrevistas com os gestores da instituição de ensino até a fase de implementação do projeto. Tais etapas foram conduzidas na ordem apresentada pela Figura 2.

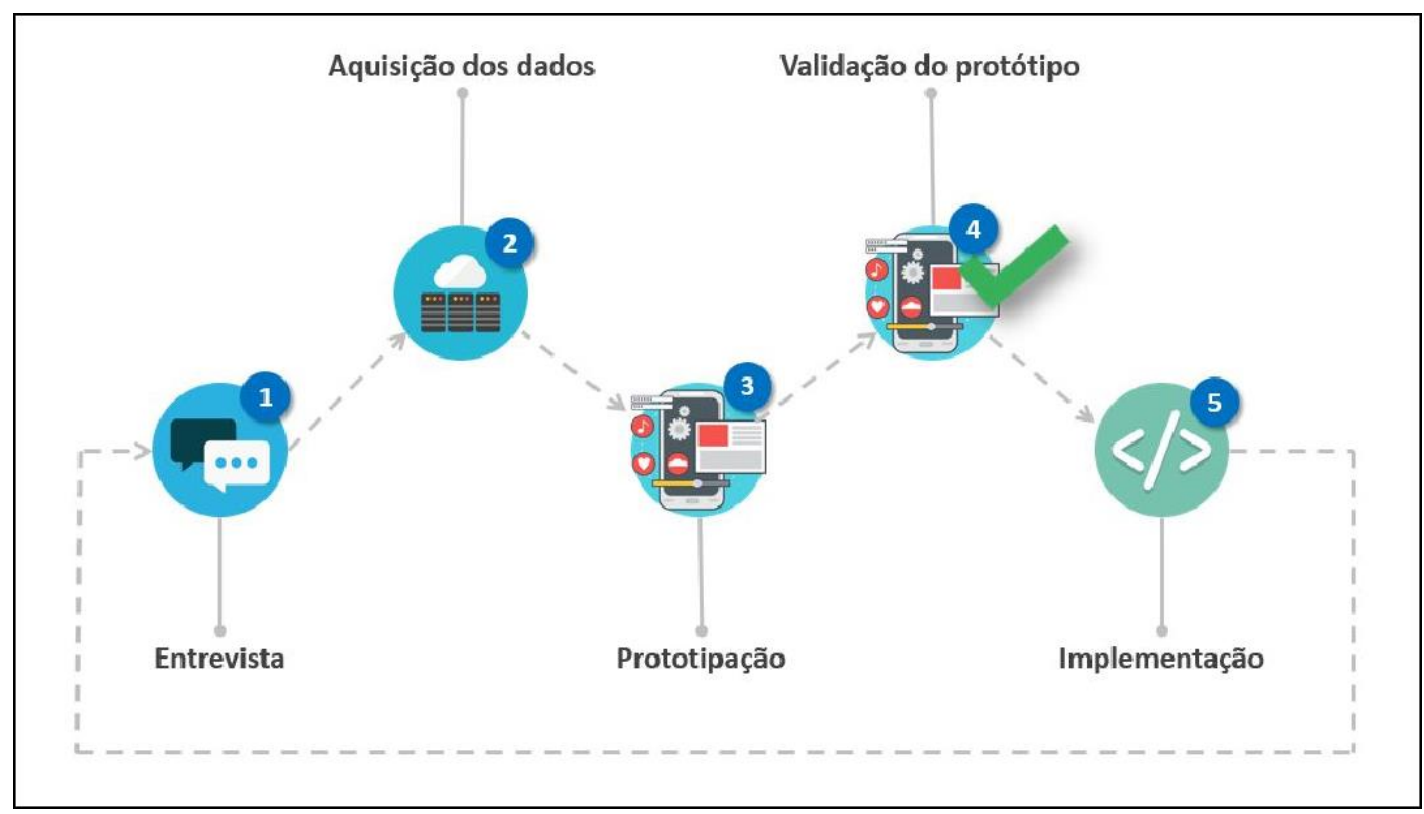

Figura 2. Metodologia

A Figura 2 ilustra a metodologia utilizada no trabalho, que consiste em 5 fases necessárias para o desenvolvimento e aquisição do resultado esperado (dashboard). Para a definição das métricas utilizadas no dashboard, foram realizadas entrevistas com especialistas de domínio (1), a Profa. Parcilene Fernandes de Brito. Parcilene Fernandes, 
que é responsável pela gestão acadêmica de uma instituição de ensino e coordenação dos cursos do Departamento de Computação. A fim de entender o contexto e obter uma visão geral do trabalho como um todo, foi realizado um estudo para verificar como os dados estão armazenados e realizada a etapa de aquisição de dados (2) presente no modelo de arquitetura por Staron (2015) (Figura 1). Para esta etapa, foram criados scripts para coleta, sumarização e armazenamentos dos dados selecionados no banco de dados da plataforma Conecta, de acordo com as métricas definidas durante as entrevistas. Para a criação dos protótipos (3) foi utilizada a ferramenta Moqups, que é uma ferramenta gratuita disponibilizada de forma online e que permite o desenvolvimento de diagramas e esboços para expor ideias sobre projetos. Após a criação dos protótipos obedecendo os conceitos elucidados no referencial teórico, esta fase teve por objetivo a realização de reuniões com a especialista de domínio para discutir sobre possíveis melhorias no dashboard e avaliar se as métricas definidas foram atendidas (4). Caso o protótipo não fosse devidamente validado, novas entrevistas (fase 1) seriam realizadas para que as devidas correções pudessem ser aplicadas ao protótipo, de forma que ele pudesse ser validado ao final desta etapa. Por fim, foi implementado (5) o dashboard para o contexto de Gestão Acadêmica. Após o processo de implementação foram realizados testes funcionais no dashboard. Em seguida, os resultados provenientes da implementação foram enviados ao Servidor de Testes da Fábrica de Software do CEULP/ULBRA, no qual a especialista de domínio teve a oportunidade de testar o software desenvolvido.

\section{Resultados}

Conforme explica Staron (2015) (Figura 5), a arquitetura do dashboard desenvolvido possui 4 etapas, sendo elas: Base de dados externas, Scripts de atualização dos dados/armazenamento, Análise de dados e Visualização. Para que os dados pudessem ser exibidos na tela do dashboard, foi definida a arquitetura cliente/servidor, com a comunicação realizada por meio de requisições HTTP.

Durante as reuniões realizadas na fase 1 (descrita na subseção 3.2), foram elaboradas métricas com o objetivo de alinhar as informações a serem apresentadas ao usuário no dashboard. Estas métricas representam totalizadores capazes de refletir os principais objetivos da organização e foram obtidas por meio de scripts que exploraram as bases de dados da plataforma Conecta. Devido a grande extensão da base, foram observadas somente as tabelas necessárias à obtenção das métricas definidas. Essa plataforma armazena as informações de forma a conservar os relacionamentos presentes entre os dados, como o vínculo entre alunos e turmas, bem como o relacionado entre turmas, disciplinas e cursos. A partir desta base, foram criados mecanismos para sumarização dos dados disponíveis para apresentá-los no dashboard de modo a atender os objetivos definidos na fase 1. Com os dados presentes na plataforma Conecta, foi possível a sumarização do conteúdo a partir das métricas definidas. Algumas delas foram: Número total de alunos, Número total de turmas, Porcentagem total de alunos frequentes em sala de aula, Porcentagem total de alunos em situação de risco em sala de aula, Porcentagem de alunos frequentes por turma, entre outras.

Foi observado que os dados são em sua maioria de natureza quantitativa. Além disso, as métricas definidas fazem referência a um curso específico presente na instituição. Para a aquisição dos dados (fase 2) e criação dos elementos gráficos exibidos na tela do dashboard, foram estabelecidos 126 cenários diferentes, que foram aplicados a cada um dos 23 cursos ativos presentes na instituição. Dessa forma, inicialmente foram realizadas 126 consultas correspondentes às métricas definidas para o primeiro curso, e em seguida foi criado um código escrito na linguagem de programação PHP para a extração das informações de forma automatizada para os cursos restantes, pois cada consulta teve que ser realizada para cada curso, totalizando mais de 2,8 mil consultas diretas realizadas na base de origem. 
A partir dos mecanismos para coleta dos dados criados, percebeu-se a necessidade de armazenamento destes totalizadores em uma nova base de dados, destinada de forma exclusiva a consulta e exibição destes resultados na tela do dashboard. O objetivo desta tarefa foi o ganho de desempenho na apresentação dos resultados na tela do dashboard, uma vez que os resultados já haviam sido previamente armazenados, o dashboard acessa os dados de forma mais rápida, em uma única consulta, sem a necessidade de execução de várias consultas para aquisição dos dados solicitados. Após as etapas de entrevista, categorização e com o conjunto de métricas definido, foi possível a elaboração dos primeiros protótipos funcionais do dashboard. A Figura 3 exibe uma das telas criadas para validação do dashboard pela especialista de domínio.

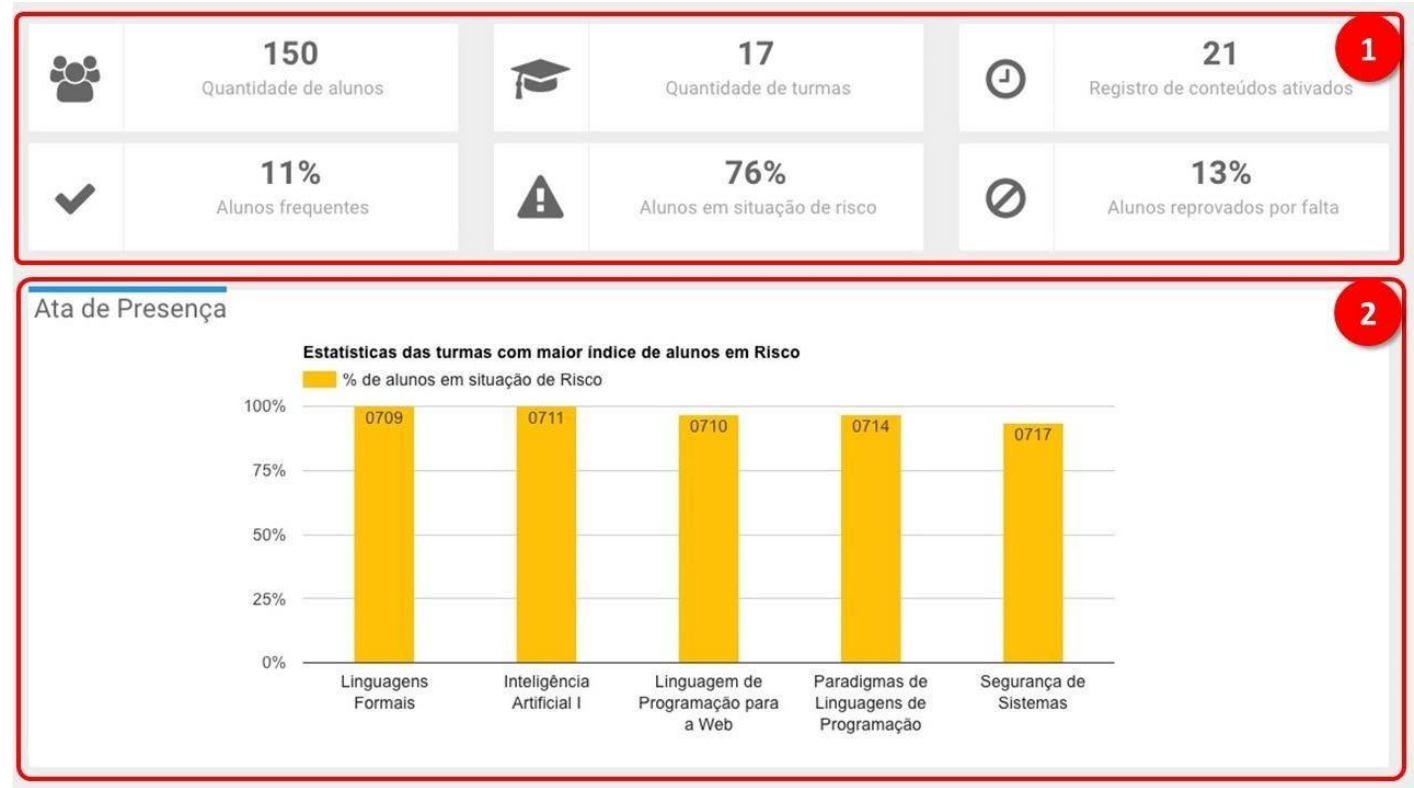

Figura 3. Protótipo de tela inicial do dashboard

A Figura 3 representa uma das telas propostas para o dashboard. A princípio é apresentado ao usuário o quantitativo total de alunos, turmas e registro de conteúdos ativados no curso (Figura 3-1), em seguida, o percentual de alunos para cada situação em relação a frequência em sala de aula (Frequentes, Em Risco ou Reprovados). Logo abaixo, é apresentada uma métrica que se refere ao percentual de alunos em situação de risco agrupados por turmas. A partir dessa informação, no segundo caso (Figura 3-2) por exemplo, o gestor poderá tomar medidas preventivas que podem refletir em um menor índice de evasão de alunos nas disciplinas do curso. A partir do protótipo criado, foi realizada a validação com a especialista de domínio (fase 4), com objetivo de adequar o protótipo criado às necessidades levantadas durante a fase de entrevistas. Para iniciar a implementação do dashboard (fase 5), foi desenvolvida uma estrutura inicial que obedece ao protótipo criado, como mostra a Figura 4.

Como o intuito de um dashboard analítico é permitir que o usuário realize operações que vão além dos dados apresentados na tela por meio de recursos de interatividade, foi criado um menu superior (Figura 4-1) para o que usuário-coordenador possa navegar entre os dois contextos, o dashboard relacionados às métricas iniciais definidas pela especialista e o questionário de percepção das provas bimestrais (que são informações adicionais, mas de relevante importância para os coordenadores). 


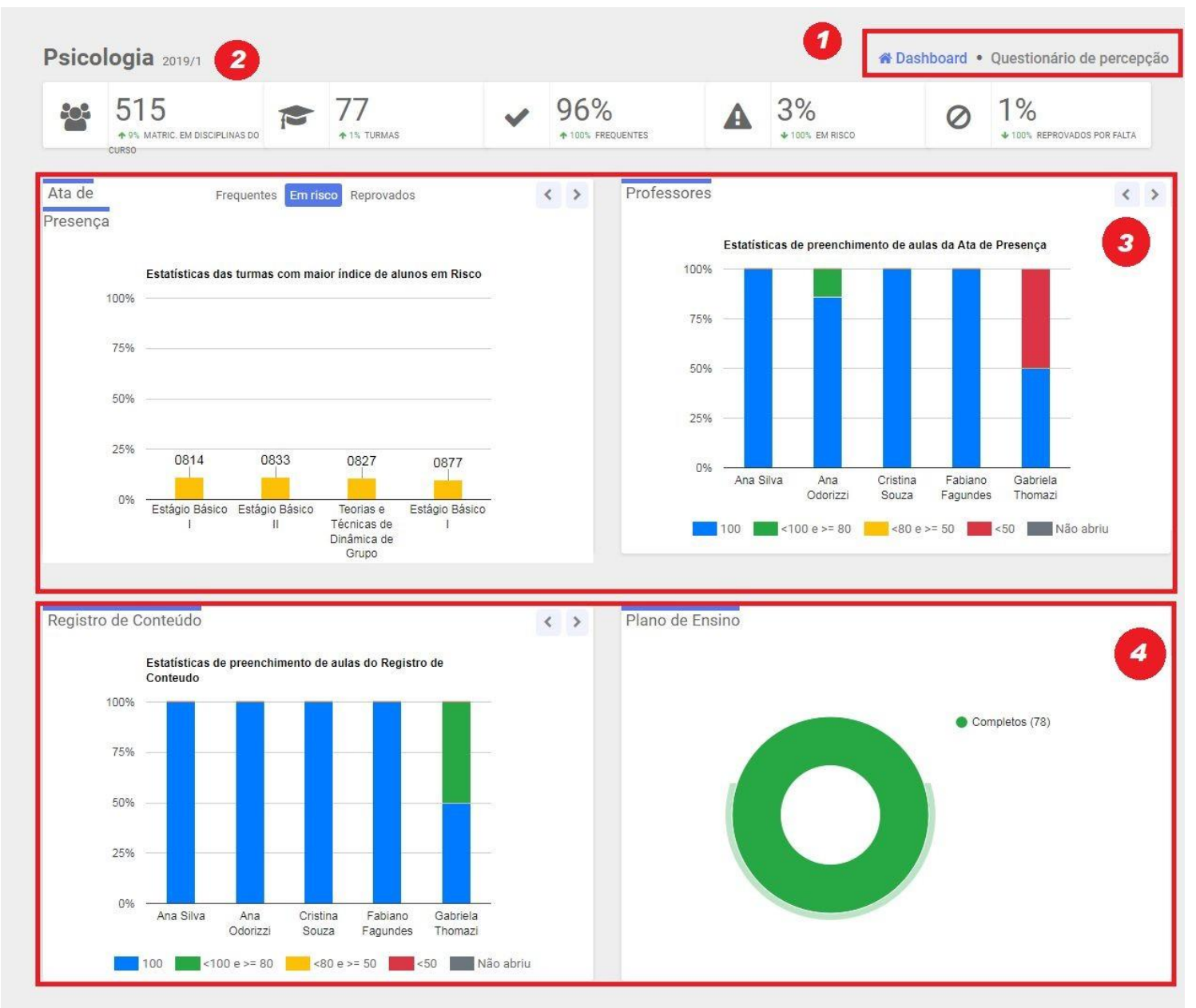

Figura 4. Parte da Tela do dashboard - Coordenação de Curso

$\mathrm{Na}$ Figura 4 é apresentada a tela inicial desenvolvida para o dashboard proposto. Conforme apresentado no protótipo do dashboard, são apresentados totalizadores de alunos, turmas e ainda o percentual de alunos para cada situação em relação a frequência em sala de aula (Frequentes, Em Risco ou Reprovados) (Figura 4 - 2). Em seguida, são apresentadas métricas referentes às funcionalidades "Professores" e "Ata de Presença" da plataforma Conecta (Figura 4 - 3), nesta última o gráfico apresentado permite que o usuário escolha um contexto específico para avaliar a situação das turmas (dos três disponíveis para a filtragem: "Frequentes", "Em Risco" ou "Reprovados").

Desta forma, é possível avaliar o percentual de assiduidade (frequência) dos alunos em sala de aula por turma. No final da tela é possível observar o gráfico que revela as estatísticas de preenchimentos da funcionalidade "Plano de Ensino" e "Registro de Conteúdo" (Figura 4 - 4), nesta última é possível que o usuário verifique a estatística de preenchimento do registro de conteúdo feita por professores a cada aula ministrada em uma turma específica.

Além do dashboard com informações relevantes para o Coordenador de Curso de uma Instituição de Ensino, também foi feito uma área especial para que a gestão acadêmica da instituição visualizasse informações sobre os cursos de uma forma comparativa, conforme pode ser observada na Figura 5 a seguir. 


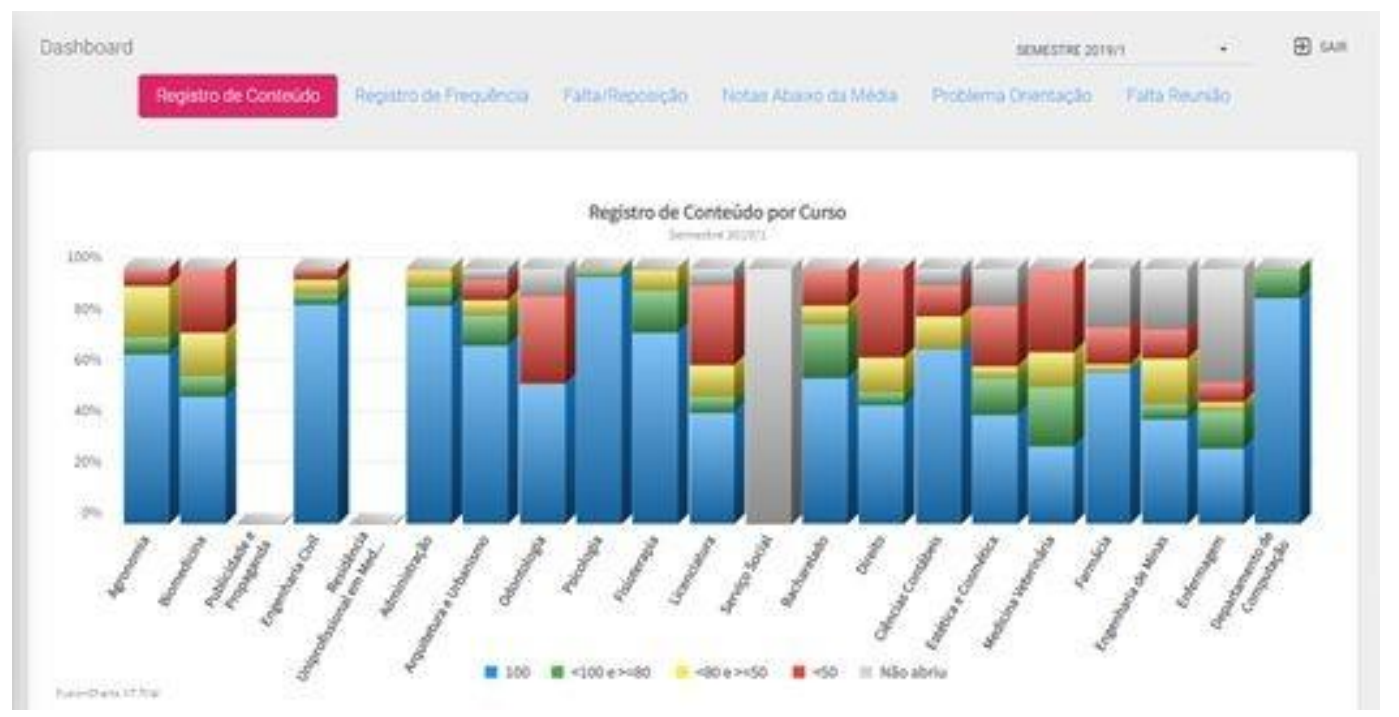

Figura 5. Tela do dashboard - Gestão Acadêmica

Conforme pode ser observado na Figura 5, as informações que constam no dashboard para o perfil Coordenador de Curso, nesta tela especial para o Gestor Acadêmico da IES (que responde pela parte acadêmica de todos os cursos), há uma visualização comparativa entre os cursos, inclusive com informações adicionais que são preenchidas pelos coordenadores, como métricas relacionadas às faltas e reposições de aulas por parte dos professores, problemas nas orientações, dentre outras.

\section{Considerações Finais}

Os recursos e técnicas apresentados na literatura voltados para criação de dashboards proporcionaram a criação de interfaces interativas e de fácil entendimento ao usuário final. A partir dos gráficos gerados foi possível analisar, por exemplo, o número de turmas com maior de índice de alunos em situação de risco (no limite da reprovação por faltas), além de uma visualização geral por parte do gestor acadêmico institucional, com totalizantes por cursos que permitiram a criação de gráficos comparativos. Isso facilita ao gestor a identificação de padrões potencialmente válidos para aplicação de um processo de intervenção na turma, a fim de estabelecer-se a causa do resultado não desejado para a situação apresentada.

Para projetos futuros, é possível agregar ainda mais informação ao dashboard ao utilizar enriquecimento por comparação para criar novas situações que envolvem métricas de diferentes semestres e não somente do semestre atual.

\section{Referências}

Buchsbaum, Paulo. Dashboards - Introdução ao conceito. [s. L.]: Fórum de Varejo, 2012. 11 p.

Duarte, João Carlos Assunção. Dashboard Visual, Uma ferramenta de Business Intelligence. 2012. 54 f. Dissertação (Mestrado) - Curso de Engenharia Mecânica, Faculdade de Engenharia da Universidade do Porto, Porto, 2012.

Few, Stephen. Information Dashboard Design: The Effective Visual Communication of Data. Sebastopol: O'reilly, 2006. 223 p.

Goldschmidt, Ronaldo; Bezerra, Eduardo. Exemplos de aplicações de data mining no mercado brasileiro: Conhecimento é fundamental para se obter diferenciais mercadológicos. Como tirar proveito de todo o patrimônio digital que vem sendo acumulado?. 2017. Disponível em: $<$ http://computerworld.com.br/exemplos-de-pulicacoesde-data-mining-no-mercado-brasileiro>. Acesso em: 30 ago. 2018.

Pauwels, K., Ambler, T., Bruce, H.C., LaPointe. P., Reibstein. D., Skiera, B.,Wierenga, B., Wiesel, T. (2009). Dashboards as a service: why, what, how, and what research is needed? J. Serv. Res.12, 175-89. 
Staron, Miroslaw. Dashboard development guide - How to build sustainable and useful dashboards to support software development and maintenance. Research Reports In Software Engineering And Management, Gotemburgo, p.1-27, 24 nov. 2015. Disponível em: <https:/gupea.ub.gu.se/bitstream/2077/41120/1/gupea_2077_41120_1.pdf $>$. Acesso em: 01 nov. 2017.

Yigitbasioglu, O.M., Velcu O. (2012). The use of dashboards in performance Management: Evidence from Sales Managers. International Journal of Digital Accounting Research, 12, $39-58$. 